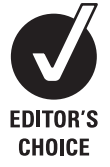
The Chester County Hospital, West Chester, Pennsylvania, USA; ${ }^{2}$ Kern Medical Center, Kern County, Bakersfield, California; ${ }^{3}$ David Geffen School of Medicine, University of California Los Angeles, Los Angeles, California, USA;

${ }^{4}$ Department of Research and Development, Medical Diagnostic Laboratories, Hamilton, New Jersey, USA

Correspondence to: Paul Walsh, Department of Emergency Medicine, Kern Medical Centre, Bakersfield, CA, 93305, USA:

yousentwhohome@gmail.com

Accepted 2 July 2009

\title{
Performance of influenza rapid antigen testing in influenza in emergency department patients
}

\author{
C Biggs, ${ }^{1}$ P Walsh, ${ }^{2,3}$ C L Overmyer, ${ }^{4}$ D Gonzalez, ${ }^{4}$ M Feola, ${ }^{4}$ E Mordechai, ${ }^{4}$ \\ $\mathrm{M}$ E Adelson, ${ }^{4} \mathrm{~K}$ T lacono ${ }^{4}$
}

\begin{abstract}
Background: The use of rapid antigen tests to triage specimens for polymerase chain reaction (PCR) testing from emergency department patients with influenza-like illness during surveillance for novel influenza viruses has been suggested.

Objective: To measure the observed sensitivity and specificity for a widely used rapid antigen test (Binax) using a PCR-based assay (Medical Diagnostic Laboratories).

Methods: Nasopharyngeal samples were taken with flocked swabs (Copan Diagnostics) from patients presenting to the emergency department of a community hospital. Samples were analysed using a rapid antigen and a PCR-based test. PCR testing was used as the criterion reference. Sensitivity and specificity were calculated for influenza and influenza A. Positive predictive values were calculated over a range of possible prevalence.
\end{abstract}

Results: Samples from 566 unique patients were tested using both methods. Sensitivity was $69.1 \%(95 \% \mathrm{Cl}$ $58.9 \%$ to $78.1 \%)$ and specificity was $97.7 \%(95 \% \mathrm{Cl}$ $95.8 \%$ to $98.8 \%)$ for the detection of any influenza and 75.3\% (95\% Cl 64.7\% to $84.0 \%$ ) and $97.8 \%$ (95\% Cl $95.9 \%$ to $98.9 \%)$, respectively, for influenza A only. The resultant positive predictive value ranges from $23 \%$ to $77 \%$ when the prevalence ranges from $1 \%$ to $10 \%$.

Conclusion: When planning early outbreak surveillance, provision of adequate PCR testing capacity rather than triaging specimens using rapid antigen testing for influenza is advisable.

Surveillance for novel influenza could be hampered by large numbers of people presenting to the emergency department with influenza-like illness, which is not in fact influenza. Surveillance is further complicated by the protean presentations of influenza. In one series, only $51 \%$ of 207 patients hospitalized with proven influenza met the Centers for Disease Control and Prevention criteria for having an influenza-like illness. ${ }^{1}$ Consequently, using rapid antigen tests for influenza to screen out non-influenza viral illness appears reasonable.

We compared the observed performance of a widely used rapid antigen test (BinaxNOW; Binax, Scarborough, Maine, USA) to a commercially available polymerase chain reaction (PCR)-based assay (MDL, Hamilton, New Jersey, USA) under typical clinical conditions.

\section{METHODS}

The study was exempt from institutional review board approval.

\section{Study design}

A blinded measurement of rapid antigen influenza test using a PCR gold standard was conducted.

\section{Study setting}

The clinical site was the emergency department of a community hospital serving 40000 adults and children annually. The rapid antigen test was performed in a clinical laboratory and the PCR assay carried out in a commercial research laboratory.

\section{Study participants}

De-identified samples for influenza testing were obtained from patients presenting between November 2006 and April 2007. Samples were obtained at the discretion of the treating emergency physician. Observed prevalence was determined by PCR assays.

\section{Materials}

Samples were obtained using nasopharyngeal nylon flocked swabs stored in universal transport medium at room temperature (UTM-RT; Copan Diagnostics, Murrieta, California, USA).

Antigen testing was performed in a clinical laboratory using the BinaxNOW methodology, an immunochromatographic assay, in accordance with the manufacturer's instructions. Samples for PCR testing were stored at $-70^{\circ} \mathrm{C}$ before shipment on dry ice to the diagnostic laboratory. This laboratory was blinded to the antigen testing results. After thawing, RNA was extracted from aliquots of UTM-RT using an automated extraction system and kit (Corbett Robotics, San Francisco, California, USA; OIAmp Viral RNA Extraction Kit, Qiagen, Valencia, California, USA) and assayed for influenza A (INF A) and influenza $B$ (INF B) by reverse transcriptase PCR.

PCR testing for INF $A$ and B was performed using an in-house developed and validated assay. Each assay was designed against a conserved viral element and validated using known positive specimens purchased from ATCC (Vanassas, Virginia, USA) or from Microbiologics (Saint Cloud, Minnesota, USA). Targeted genetic elements were PCR amplified and subcloned into PCR TOPO 2.1 vectors to serve as assay optimisation standards.

\section{Statistical analysis}

We estimated sensitivity, specificity and positive and negative predictive values (PPV and NPV, respectively) for the antigen test using PCR as the gold standard. Prevalence was determined from 
Table 1 PCR and rapid immunochromatographic antigen testing results for all included samples

\begin{tabular}{llcc}
\hline & \multicolumn{2}{l}{ Rapid antigen test } & \\
\cline { 2 - 3 } PCR influenza & Positive & Negative & Total \\
\hline Positive & 67 & 30 & 97 \\
Negative & 11 & 458 & 469 \\
Total & 78 & 488 & 566 \\
\hline
\end{tabular}

PCR, polymerase chain reaction.

PCR results. Age groups were stratified as follows: 0-24 months (infants and toddlers), 2-18 years (children) and older 18 years (adults). We calculated PPV and NPV for the rapid antigen test for a range of possible prevalence of influenza. We derived PPV and NPV for potential prevalence that can be obtained when surveillance is being performed. In a secondary analysis test, characteristics and predictive values were calculated for INF A only. Statistical analysis was performed using STATA 10.1 software (Statacorp LP, College Station, Texas, USA).

\section{RESULTS}

Samples from 566 unique patients were tested using both methods. A disproportionate number $(165 / 564,29 \%)$ of the tests was performed in infants and toddlers. Among older children aged 2 to 18 years $(118 / 564,21 \%$, of the sample), the median age was 6 years (interquartile range $3-11)$. Two hundred and eighty-one $(281 / 564,50 \%)$ were adults (age data missing in 2 of 566).

The prevalence of influenza by PCR was $17.1 \%$ (95\% confidence interval (CI) $14.0 \%$ to $20.5 \%$ ) overall and $15 \%$ (95\% CI $12.0 \%$ to $18.2 \%$ ) for type A only. Among infants and toddlers, prevalence was $6.7 \%$ (95\% CI $3.4 \%$ to $11.6 \%$ ), in children $2-18$ years was $30.5 \%$ (95\% CI $22.3 \%$ to $39.7 \%$ ) and in adults was $17.8 \%$ (95\% CI $13.5 \%$ to $22.8 \%$ ).

The PCR and immunochromatographic rapid antigen results are shown in table 1 . Sensitivity of the rapid antigen test was $69.1 \%$ (95\% CI $58.9 \%$ to $78.1 \%$ ) and specificity was $97.7 \%$ (95\% CI $95.8 \%$ to $98.8 \%$ ). The positive predictive value for the antigen test over a range of possible prevalence is shown in fig 1 . The BinaxNOW assay performed better for INF A (see table 2), with a sensitivity of $75.3 \%$ (95\% CI $64.7 \%$ to $84.0 \%$ ) and a specificity of $97.7 \%$ (95\% CI $95.9 \%$ to $98.9 \%$ ), than for INF B (sensitivity $20 \%, 95 \%$ CI $4.3 \%$ to $48.1 \%$; specificity $100 \%$, $95 \%$

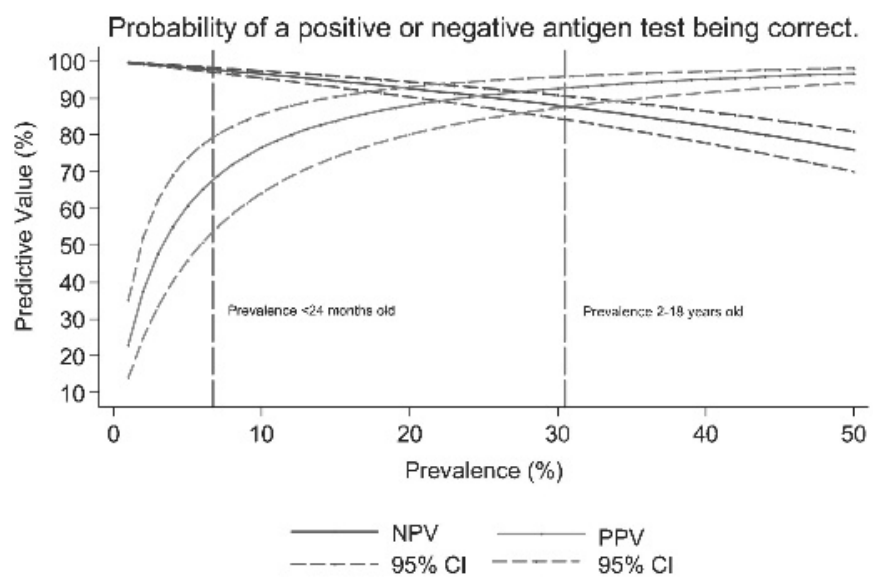

Figure 1 Relationship between prevalence and predictive values for influenza rapid antigen test. NPV, negative predictive value; PPV, positive predictive value.

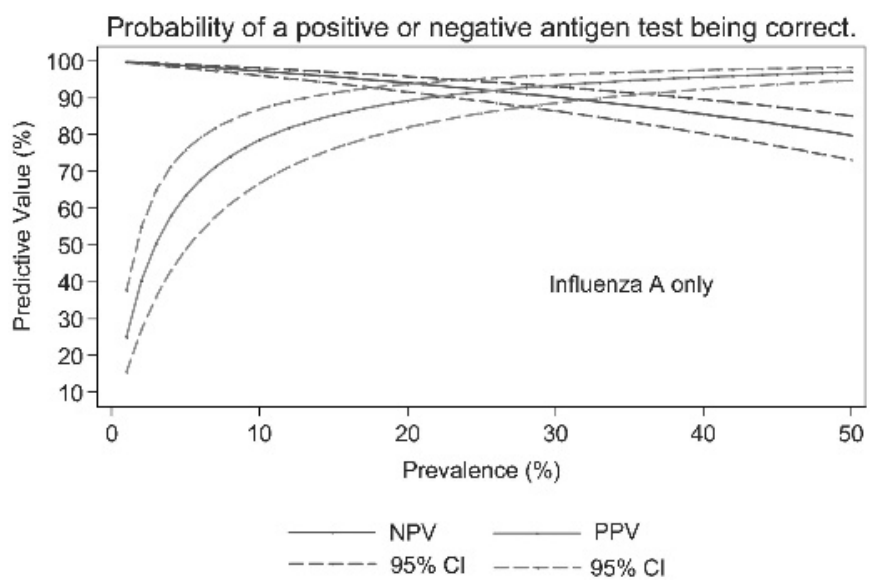

Figure 2 Relationship between prevalence and predictive values for INF A only using the antigen test. INF A, influenza A; NPV, negative predictive value; PPV, positive predictive value.

CI $99.3 \%$ to $100 \%)$ ) The implications for predictive values for detection of INF A are shown in fig 2.

\section{DISCUSSION}

Although in the event of an actual pandemic, viral testing may be scaled back, extensive testing does need to be performed in the early surveillance phase. ${ }^{2}$ Emergency physicians and administrators might reasonably intuit that rapid antigen testing would allow triaging of specimens for PCR testing. Our results do not support this strategy. The predictive values of antigen testing depend on both the actual sensitivity and specificity of the antigen test under clinical conditions and the prevalence of influenza at the time the test is performed. When prevalence is low, for example, $5 \%$, a positive test will be a true positive in only $61 \%$ of the cases. Conversely, when prevalence is high, for example, $50 \%$, a negative test will be a false negative in $24 \%$ of the cases.

This is unfortunate because the rapid antigen test can be completed in about 20 minutes following "receipt at the hospital laboratory. The turnaround time at the commercial laboratory where PCR was performed is 24 to $36 \mathrm{~h}$, although times of $5.5 \mathrm{~h}$ for influenza PCR have been reported. ${ }^{3}$

The difference in prevalence we observed in seasonal influenza between infants and toddlers and older children likely reflects both different indications for ordering the test and different manifestations of influenza between these groups. In febrile infants, viral antigen testing may be used to minimize evaluations for bacterial illness. ${ }^{45}$ Influenza in infants may present as bronchiolitis ${ }^{6-8}$ or as other febrile illnesses ${ }^{9}$ that are more difficult to evaluate because these children are preverbal. Emergency physicians who adopt this strategy and rely on antigen testing need to consider both the prevalence of the virus and the characteristics of the test that they are using.

Table 2 Performance of PCR and rapid immunochromatographic antigen tests for INF A only

\begin{tabular}{llcc}
\hline \multirow{2}{*}{ PCR INF A only } & \multicolumn{2}{l}{ Rapid antigen testing } & \\
\cline { 2 - 3 } & Positive & Negative & Total \\
\hline Positive & 64 & 21 & 85 \\
Negative & 11 & 470 & 481 \\
Total & 75 & 491 & 566 \\
\hline
\end{tabular}

INF A, influenza A; PCR, polymerase chain reaction. 
In contrast, it seems likely that the ordering of influenza antigen testing in older children and adolescents was based on the firmer clinical suspicion that a direct history allows. These situations demonstrate how even when not in a period of heightened surveillance prevalence and test predictive values can vary between patient groups.

Previous influenza pandemics have been characterized by a shift in mortality toward younger age groups. ${ }^{10}{ }^{11}$ This trend has also emerged with the recent novel swine H1N1 influenza pandemic ${ }^{12}$ and may inform screening criteria in a future wave. A comparison of that age profile and our seasonal influenza one are shown in Appendix 1.

Our results are substantially poorer than those reported in the package insert for the rapid antigen test. However, other investigators have had similar findings to ours with the BinaxNOW assay in children ${ }^{13}$, and adults and children ${ }^{14}$ using viral culture as the gold standard.

Obtaining PCR in a timely manner may be difficult outside of academic settings. In the context of pandemic surveillance, a strategy of home quarantine pending PCR results may be considered. The decision to use a negative rapid antigen result to avoid home quarantine will depend on, among other factors, the pathogenicity of the virus and its prevalence. When prevalence is very low, even a technically unreliable test can have a high negative predictive value.

\section{LIMITATIONS}

Sensitivity and specificity, although theoretically stable, may in fact vary if the populations differ or, through a spectrum effect, if a disease is being tested for early in its course. Consequently, it is possible that sensitivity may be somewhat lower if the patients submitted to surveillance are less sick than those who would ordinarily have the test ordered in clinical practice.

We did not use a third method (eg, direct immunofluorescence or viral culture) to resolve discordant rapid antigen and PCR results, instead treating PCR as the criterion reference. The former of these is technician dependent, and the latter not likely to be widely used, particularly for highly pathogenic strains. Prior comparison of immunochromatographic testing, cytospinenhanced direct immunofluorescence and PCR has shown PCR to be the testing method of choice. ${ }^{15}$ Similar results have been obtained comparing immunochromatographic tests, culture and PCR. ${ }^{16}$

We tested a single rapid antigen immunochromatographic test, and extrapolation to other rapid antigen tests is problematic. However, other investigators have found the BinaxNOW kit to perform similarly to, or better than, other commonly used rapid tests. ${ }^{14} 1718$

The lack of clinical information available to us about these patients limits our results to the characteristics of the tests performed. We can only speculate as to the clinical reasoning for testing for influenza in these patients; however, the spectrum of clinical presentations of influenza is well described. ${ }^{189} 1119$

\section{CONCLUSION}

When planning early low prevalence outbreak surveillance as distinct from clinically indicated testing, provision of adequate PCR testing capacity rather than triaging specimens using rapid antigen testing for influenza is advisable.

Acknowledgements: The authors would like to acknowledge Thomas Purcell MD and Mark Lowen for their assistance.
Competing interests: None declared.

Provenance and peer review: Not commissioned; externally peer reviewed.

\section{REFERENCES}

1. Babcock HM, Merz LR, Fraser VJ. Is influenza an influenza-like illness? Clinical presentation of influenza in hospitalized patients. Infect Control Hosp Epidemiol 2006:27:266-70.

2. Public Health Agency of Canada. The Canadian pandemic influenza plan for the health sector-annex N. 2009.

3. Zitterkopf NL, Leekha S, Espy MJ, et al. Relevance of influenza A virus detection by PCR, shell vial assay, and tube cell culture to rapid reporting procedures. J Clin Microbiol 2006:44:3366-7.

4. Iyer SB, Gerber MA, Pomerantz WJ, et al. Effect of point-of-care influenza testing on management of febrile children. Acad Emerg Med 2006;13:1259-68.

5. Smitherman HF, Caviness AC, Macias CG. Retrospective review of serious bacterial infections in infants who are 0 to 36 months of age and have influenza $A$ infection. Pediatrics 2005;115:710-8.

6. Sung RY, Chan PK, Tsen T, et al. Identification of viral and atypical bacterial pathogens in children hospitalized with acute respiratory infections in Hong Kong by multiplex PCR assays. J Med Virol 2009;81:153-9.

7. Mansbach JM, McAdam AJ, Clark S, et al. Prospective multicenter study of the viral etiology of bronchiolitis in the emergency department. Acad Emerg Med 2008;15:111-8.

8. Friedman MJ, Attia MW. Influenza a in young children with suspected respiratory syncytial virus infection. Acad Emerg Med 2003;10:1400-3.

9. Chiu SS, Tse CY, Lau YL, et al. Influenza A infection is an important cause of febrile seizures. Pediatrics 2001;108:E63.

10. Andreasen V, Viboud C, Simonsen L. Epidemiologic characterization of the 1918 influenza pandemic summer wave in Copenhagen: implications for pandemic control strategies. J Infect Dis 2008;197:270-8.

11. Miller MA, Viboud C, Balinska M, et al. The signature features of influenza pandemics - implications for policy. N Engl J Med 2009;360:2595-8.

12. Novel Swine-Origin Influenza A (H1N1) Virus Investigation Team. Emergence of a novel swine-origin influenza A (H1N1) virus in humans. $N$ Engl J Med 2009;360:2605-15.

13. Cruz AT, Cazacu AC, Greer JM, et al. Rapid assays for the diagnosis of influenza A and $B$ viruses in patients evaluated at a large tertiary care children's hospital during two consecutive winter seasons. J Clin Virol 2008;41:143-7.

14. Hurt AC, Alexander R, Hibbert J, et al. Performance of six influenza rapid tests in detecting human influenza in clinical specimens. J Clin Virol 2007;39:132-5.

15. Landry ML, Cohen S, Ferguson D. Real-time PCR compared to Binax NOW and cytospin-immunofluorescence for detection of influenza in hospitalized patients. J Clin Virol 2008:43:148-51

16. Rahman M, Vandermause MF, Kieke BA, et al. Performance of Binax NOW flu $A$ and $B$ and direct fluorescent assay in comparison with a composite of viral culture or reverse transcription polymerase chain reaction for detection of influenza infection during the 2006 to 2007 season. Diagn Microbiol Infect Dis 2008;62:162-6.

17. Landry ML, Cohen S, Ferguson D. Comparison of Binax NOW and directigen for rapid detection of influenza A and B. J Clin Virol 2004;31:113-5.

18. Smit M, Beynon KA, Murdoch DR, et al. Comparison of the NOW influenza A \& B NOW flu $A, N O W$ flu $B$, and directigen flu $A+B$ assays, and immunofluorescence with viral culture for the detection of influenza $A$ and $B$ viruses. Diagn Microbiol Infect Dis 2007:57:67-70.

19. Silvennoinen H, Peltola V, Lehtinen $\mathbf{P}$, et al. Clinical presentation of influenza in unselected children treated as outpatients. Pediatr Infect Dis J 2009;28:372-5.

Appendix 1. Comparison of the age profile in novel swine influenza and our sample of seasonal influenza

\begin{tabular}{lll}
\hline Age category & $\begin{array}{l}\text { H1N1 novel swine flu* } \\
(\mathbf{n}=\mathbf{6 4 2})(\%)\end{array}$ & $\begin{array}{l}\text { Sample studied } \\
(\mathbf{n}=\mathbf{9 7 / 5 6 6 )}(\%)\end{array}$ \\
\hline$<24$ months & 3 & 11 \\
$2-4$ years & 5 & 10 \\
$5-9$ years & 12 & 14 \\
10-18 years & 40 & 12 \\
19+ years & 40 & 52 \\
\hline
\end{tabular}

*Denominator not reported. From Andreasen et al. ${ }^{10}$

${ }^{\dagger}$ Age missing in two. Less than $100 \%$ because of rounding. 